\title{
IMPACTO DE LA VARIABILIDAD CLIMÁTICA EN EL BALANCE HÍDRICO DE LA CIÉNAGA DE LAS MACANAS
}

\author{
Serra, Yvanna \\ Facultad de ingeniería civil UTP \\ Panamá, Panamá \\ yvanna.serra@utp.ac.pa \\ Santamaria, Andrea \\ Facultad de ingeniería civil UTP \\ Panamá, Panamá \\ andrea.santamaria@utp.ac.pa \\ De León, Conrado \\ Centro Regional para el Hemisferio Occidental CREHO Ramsar \\ Panamá, Panamá \\ conrado.deleon09@gmail.com \\ Fábrega Duque, José \\ Centro de investigaciones hidráulicas e hidrotecnias, UTP \\ Miembro del Sistema nacional de investigación de Panamá (SNI) \\ Panamá, Panamá \\ https://orcid.org/0000-0003-1536-0386
}

\begin{abstract}
The "Cienega de las Macanas" is the largest wetland system in the central provinces of Panama. The health of the ecosystem and the related economic activities surrounding this swamp are very dependent on the annual precipitation in this area. It was severely affected by the droughts of 2015 where it almost completely dried up. This situation is extremely serious because once a wetland dries up, it is difficult and expensive to bring it back to normal conditions. Therefore, hydrological studies are necessary to prevent drought scenarios and understand the behavior of these water bodies. This analysis quantifies how climate change has affected meteorological variables such as temperature and precipitation. It also analyzes how the change of these variables affect the water balance of the swamp, taking precipitation and evapotranspiration as study parameters. Temperature and precipitation increased $0.54 \mathrm{C}$ and $204 \mathrm{~mm}$ for a study period of 22 years. In the water balance, it was
\end{abstract}


possible to evaluate that the drought years were 2000, 2015 and 2019, which would explain why the swamp was about to dry up in 2015 , showing that there is a strong contribution by precipitation to the water balance. Our goal is to use this new information, to create effective strategies to prevent the degradation of these ecosystems.

Keywords: Wetland, Panama, climate change, hydrology, water balance.

\section{Resumen}

La ciénaga de las macanas es el mayor sistema de humedales en las provincias centrales de Panamá. Las actividades económicas y la salud de ecosistema que rodea la ciénaga es muy dependiente de la precipitación anual en esta área, la misma fue duramente afectada por las sequías del 2015 en donde casi llega a secarse por completo. Esta situación es sumamente grave debido a que una vez se seca un humedal es difícil y costoso que vuelva a la normalidad. Los estudios hidrológicos son necesarios para prevenir escenarios de sequía y entender el comportamiento de estos cuerpos de agua. Este análisis cuantifica como el cambio climático ha afectado las variables meteorológicas tales como temperatura y precipitación, y a su vez analiza como el cambio de estas variables afectan al balance hídrico de la ciénaga, tomando como parámetros de estudios la precipitación y la evapotranspiración. La temperatura y la precipitación vieron aumentos de 0.54C y $204 \mathrm{~mm}$ para un período de estudio de 22 años. En el balance hídrico se pudo evaluar que los años de sequía fueron el 2000, 2015 y 2019, lo cual explicaría porque le ciénaga estuvo a punto de secarse en el año 2015 demostrando que hay un fuerte aporte por parte de la precipitación al balance hídrico. Nuestra meta es utilizar esta nueva información para crear estrategias efectivas que prevengan la degradación de estos ecosistemas.

Palabras claves: Humedal, Panamá, Cambio climático, Hidrología, Balance hídrico.

\section{INTRODUCCIÓN}

El humedal de la ciénaga de las macanas, está ubicado en el corregimiento de El Rincón, en el distrito de Santa María, en Herrera, Panamá [1]. Se encuentra en la parte baja de la cuenca del rio Santa María, contando con un área de drenaje total de 3,400.63 Km2 desde su nacimiento hasta la desembocadura en el mar (Bahía de Parita). La longitud del río es de 
$168 \mathrm{Km}$. La parte baja de esta cuenca se vincula con el sistema costero marino de la Bahía de Parita, manglares y actividades turísticas. El potencial y la conservación de este sistema depende de un manejo adecuado de las tierras en las partes alta y media de la cuenca [2].

La ciénaga es el mayor sistema de humedales en las provincias centrales, y es un punto clave para las aves migratorias procedentes de Estados Unidos y Canadá, por estos motivos la ciénaga fue declarada como área de recursos múltiples contando con una extensión a 857 hectáreas con $6102 \mathrm{~m}^{2}$ el 19 de Julio del 2016 [1].

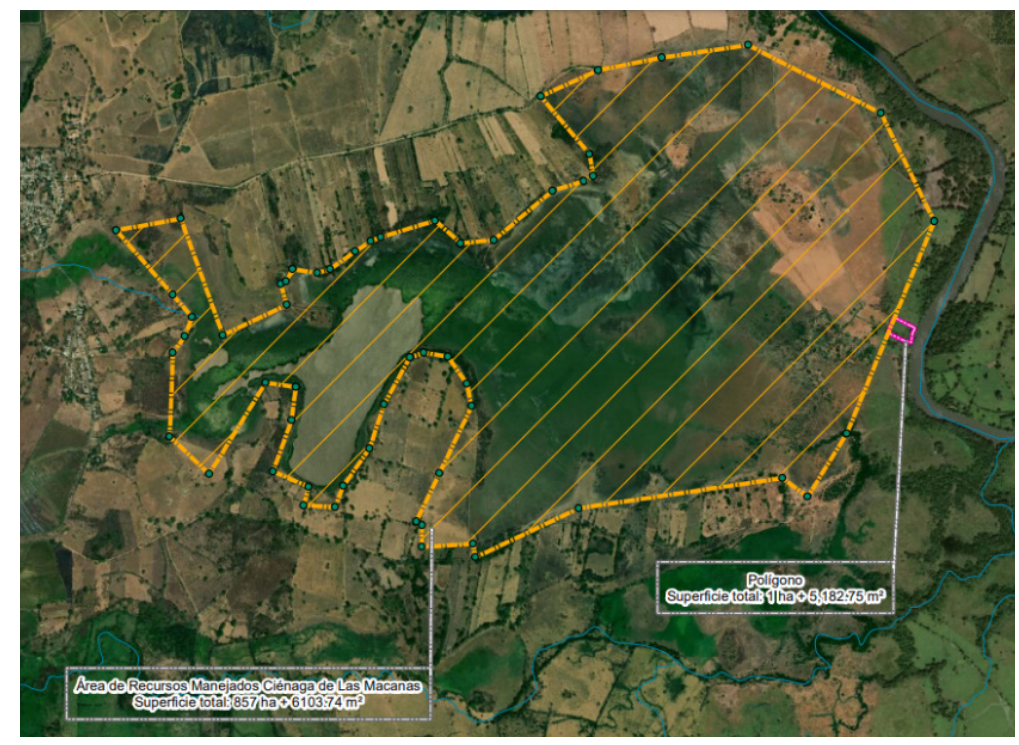

Fig. 1.Polígono del área protegida de las Ciénaga de las Macanas.

En el año 2015 tras un largo periodo de sequía el espejo de agua de la ciénaga de las Macanas casi desaparece por completo, afectando gravemente a la fauna y flora de la zona [3]. Esta situación no se había visto anteriormente, lo que puede ser un indicio de afectaciones propias del cambio climático. Debido a estos cambios extremos en el balance hídrico de la ciénaga, nuestro objetivo en esta investigación es entender como el cambio climático afecta a las variables meteorológicas y como estas a su vez afectan el balance hídrico de la ciénaga.

\section{MÉTODO}

\section{A. ÁREA DE ESTUDIO}

Se definió que el área de estudio sería el área de drenaje de la ciénaga de las macanas, delimitándose como una cuenca hidrográfica, yd tomando como punto de desembocadura la unión del afluente de los Toritos con el río Escotá. La topografía del terreno fue descargada de la página oficial del Tommy Guardia y las curvas de nivel fueron exportadas al programa 
de Austodesk Civil 3D, el cual nos permitió crear una superficie más realista del terreno y de esa forma delimitar de forma más precisa la microcuenca.

\section{B. FUENTE DE DATOS}

Los datos de precipitación se obtuvieron del pluviómetro de ETESA ubicado en Divisa y contenía datos históricos que iban de 1990 hasta 2012 y del pluviómetro de MIAMBIENTE localizado en El Rincón, el cual contenía información desde 1997 hasta el presente. Para la evapotranspiración se utilizó la ecuación de Penman-Monteith la cual necesita data de temperatura máxima y mínima, velocidad promedia del viento y humedad relativa. Para su cálculo del año 1990 al 2012 se utilizó data de la estación meteorológica de Divisa (ETESA), a partir del año 2012 hasta el 2019 se utilizó data de la NASA, los parámetros meteorológicos se derivan del modelo de asimilación GMAO MERRA-2 de la NASA y GEOS 5.12.4 FP-IT. MERRA-2 es una versión del Sistema de Asimilación de Datos del Sistema de Observación de la Tierra Goddard (GEOS) de la NASA [4].

\section{RESULTADOS}

\section{A. ÁREA DE DRENAJE}

Tras analizar la topografía del terreno en civil 3D se pudo delimitar un área de escorrentía de 2150 ha, que se extiende en el norte hasta el rio Santa María, y en el sur con el río Escotá. Dentro de esta área de drenaje se encuentra la comunidad de El Rodeo la cual se encuentra al Noroeste de esta. El área de drenaje es mucho mayor que el área protegida ( 857 ha).

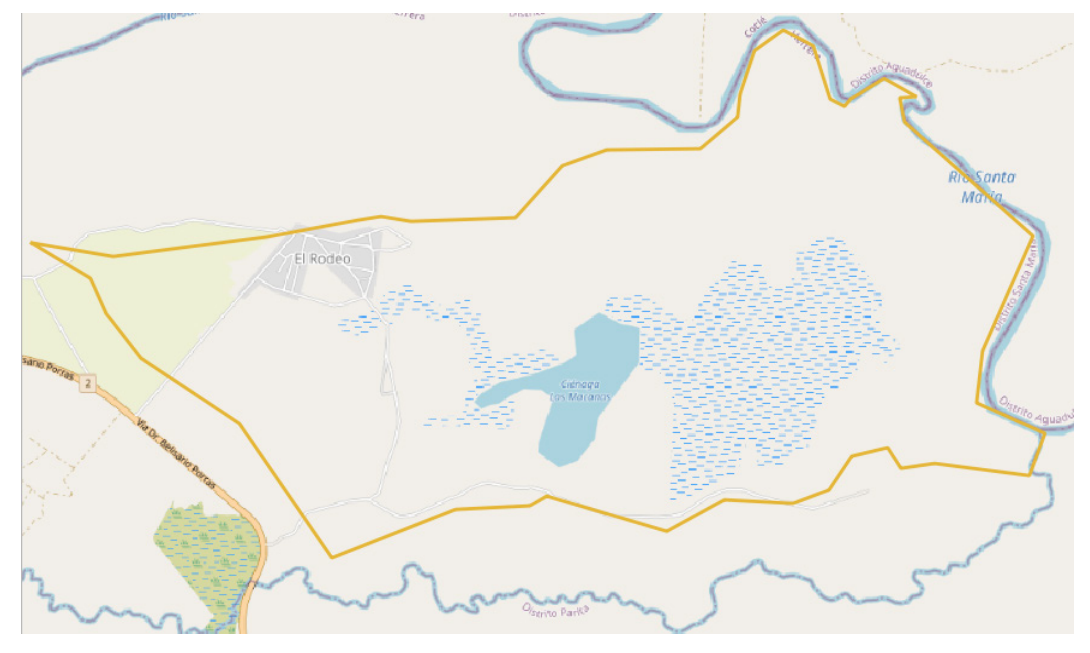

Fig. 2 Esquema del área de drenaje. 


\section{B. ANÁLISIS DE LA DATA HIDROCLIMÁTICA}

Los datos analizados de la estación meteorológica de Divisa (1990-2012) muestran una línea de tendencia de aumento de temperatura (fig. 3-a), con un aumento total promedio de 0.54 C. Los datos de precipitación estudiados corresponden a Divisa (1990-1997) y del año (1997-2012) se utilizan los datos del pluviómetro del Rincón por ser más cercanos a la Ciénaga. En conjunto estos datos muestran una línea de tendencia positiva (fig. 3-b), con un aumento de $204.4 \mathrm{~mm}$ para el período de 22 años analizados. Finalmente la evapotranspiración, para la cual se utilizaron los datos de la estación de Divisa (1990-2012) se muestra bastante constante (fi. 3-c). Para esta figura (3-c), cabe resaltar el descenso excepcional observado en el año 1997 donde se tuvo un valor de $665 \mathrm{~mm}$, comparado a valores cercanos a los $1600 \mathrm{~mm}$ que se observaron en los otros años.

b)

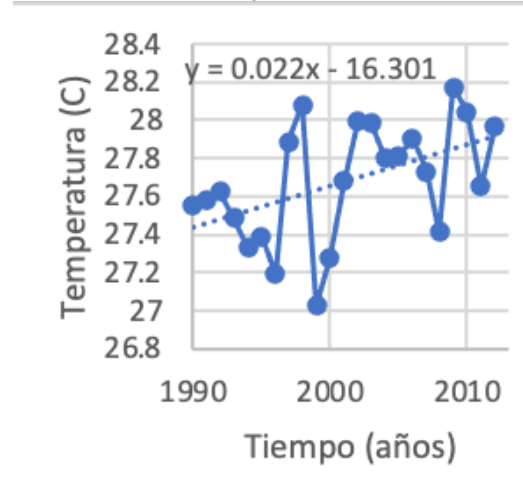

c)

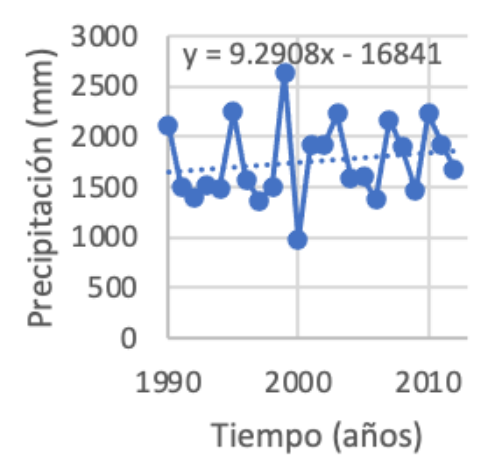

Fig. 3 Líneas de tendencia en temperatura (a), precipitación (b), y evapotranspiración (c) para la estación meteorológica de Divisa desde 1990 hasta 2012.

\section{BALANCE HÍDRICO}

Se utilizaron los datos descritos en la sección de método para calcular el balance hídrico (1990-2019). En la mayoría de los años se puede ver una evapotranspiración superior a la precipitación. La precipitación promedio anual dio un total de $1718 \mathrm{~mm}$, con sequías para los años 2000, 2015 y 2019, teniendo una precipitación por debajo de 1288mm. Mientras que los años considerados con exceso de precipitación fueron 1995, 1999, 2003, 2007 y 2010 (precipitaciones superiores a $2148 \mathrm{~mm}$ ). La evapotranspiración promedio dio como resultado $1573.7 \mathrm{~mm}$. 


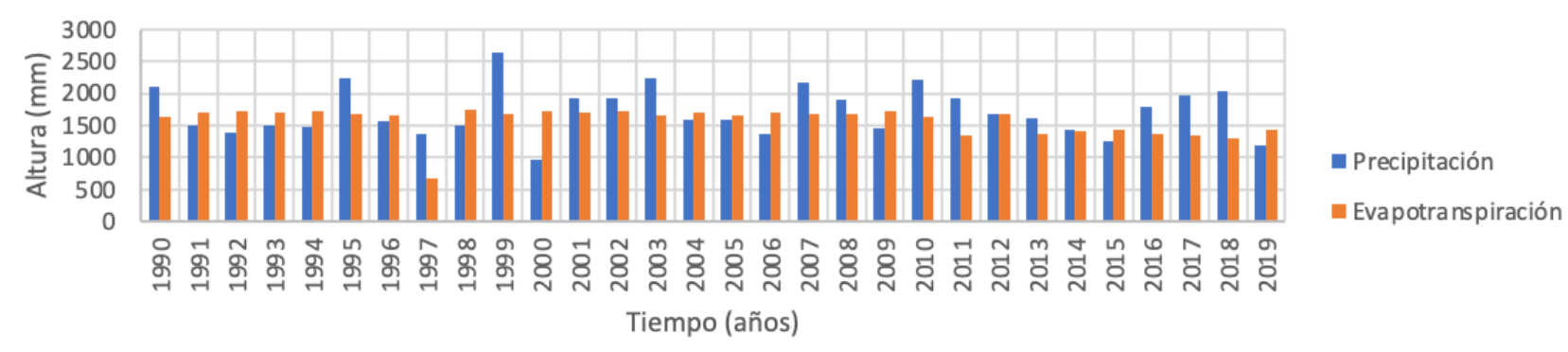

Fig. 4. Componentes del balance hídrico tomando en cuenta precipitación y evapotranspiración.

\section{CONCLUSIONES}

Los factores climáticos han presentado variaciones importantes durante el período que cubre este estudio (1990-2019). Estas variaciones pueden estar asociadas al cambio climático especialmente, en lo relativo a la temperatura. Esta situación contrasta con la poca variación en los valores de evapotranspiración. La evapotranspiración muestra una medida anormal en el año 1997 la cual pudo ocurrir por algún fallo de la estación meteorológica en ese año.

En el balance hídrico se puede notar que por lo general la precipitación suele ser menor a la evapotranspiración, y que suele haber años excepcionales de lluvia cada cuatro o cinco años. Como conclusión preliminar podemos decir que las lluvias interanuales parecen tener un efecto importante en la recarga de agua a la ciénaga cada cuatro o cinco años.

La sequía del 2015 pudo ser causada por una combinación de un año con poca precipitación y porque no ocurrió ningún evento de precipitación extraordinaria desde el año 2010. Pese a que el año 2019 hubo menos precipitación, la misma no afecto tanto a la ciénaga como en el año 2015, lo mismo pudo ocurrir debido a que en el 2018 si se registró unos altos niveles de precipitación,

Finalmente, hace falta evaluar la influencia de las aguas subterráneas y la escorrentía para tener un análisis más fiable del balance hídrico y conocer cuál es la fuente principal de agua de la Ciénaga de las Macanas

\section{Referencias}

[1] “Resolución DAPVS-0007-2016." 2016.

[2] Ministerio de ambiente "Plan de menejo integral de la parte alta, media y baja de la cuenca del rio Santa María" julio 2009 [En línea] https://cuencas.miambiente.gob.pa/wp-content/ uploads/2020/08/Plan-de-Manejo-Integrado-de-la-Cuenca-del-r\%C3\%ADo-Santa-Mar\%C3\%ADa.pdf

[3] TVN “La Ciénaga de las Macanas se seca por falta de lluvia”19 de mayo del 2015 [En línea] https:// www.tvn-2.com/nacionales/Cienaga-Macanas-seca-falta-lluvias 0 4212328751.html

[4] Nasa "Power data Acces"[En Línea] https://power.larc.nasa.gov/beta/ 


\section{Autorización y Licencia CC}

Los autores autorizan a APANAC XVIII a publicar el artículo en las actas de la conferencia en Acceso Abierto (Open Access) en diversos formatos digitales (PDF, HTML, EPUB) e integrarlos en diversas plataformas online como repositorios y bases de datos bajo la licencia CC:

Attribution-NonCommercial-ShareAlike 4.0 International (CC BY-NC-SA 4.0) https://creativecommons. org/licenses/by-nc-sa/4.0/.

Ni APANAC XVIII ni los editores son responsables ni del contenido ni de las implicaciones de lo expresado en el artículo. 